Décadrages Décadrages

cinéma, à travers champs Cinéma, à travers champs

4-5 | 2005

David Lynch

\title{
Twin Peaks. La loi des séries
}

\section{François Bovier et André Chaperon}

\section{(2) OpenEdition}

Journals

Édition électronique

URL : https://journals.openedition.org/decadrages/575

DOI : 10.4000/decadrages.575

ISSN : 2297-5977

\section{Éditeur}

Association Décadrages

\section{Édition imprimée}

Date de publication : 10 avril 2005

Pagination : 18-31

ISBN : 978-2-9700582-1-2

ISSN : 2235-7823

Référence électronique

François Bovier et André Chaperon, «Twin Peaks. La loi des séries », Décadrages [En ligne], 4-5 | 2005, mis en ligne le 29 avril 2013, consulté le 28 mars 2022. URL : http://journals.openedition.org/ decadrages/575; DOI : https://doi.org/10.4000/decadrages.575

(B Décadrages 


\section{Twin Peaks. La loi des séries}

par François Bovier et André Chaperon

1 Notre analyse du générique est basée sur la version française de la série éditée en vidéo, où le pilote n'existe pas en tant que tel. Pour une analyse du pilote, $c f$. Damien Detcheberry, "Les choses derrière les choses", in Eclipses, $\mathrm{n}^{\circ} 34,2002$, p. 92-95.

Une mésange, en plan rapproché, se tient sur une branche d'arbre (fig. 1). Une aciérie, en plan d'ensemble, est cadrée en légère plongée (fig. 2). Les mécanismes dentés et en mouvement de l'aciérie, projetant quelques étincelles, sont ressaisis à travers deux plans de détail (fig. 3-4). La caméra cadre en plan d'ensemble fixe une route qui s'enfonce dans une forêt; sur la droite, un panneau de bienvenue annonce la localité de Twin Peaks (fig. 5). La caméra cadre une chute d'eau en plan d'ensemble, puis panote vers le bas en diagonale, de gauche à droite (fig. 6). Un autre panoramique court au fil de l'eau, dans la même direction mais cette fois horizontalement (fig. 7). Le passage d'un plan à l'autre est systématiquement ponctué par un fondu enchaîné. Ce rythme apaisé, ce mouvement étale et cet invisible raccordement entre les espaces sont encore soulignés par un leitmotiv musical qui rapidement acquiert le statut de marque de signature de la série: une scie musicale immerge le télé-spectateur dans l'univers de la fiction. Ainsi débutent les trente épisodes de la série TV Trwin Peaks (1990-1991)1․ Commençons par déplier les dénotations et connotations de ces plans "génériques».

\section{L'entrée du téléspectateur dans la fiction}

L'opposition entre le motif de l'oiseau (fig. 1) et le milieu de l'aciérie (fig. 2) renvoie à un rapport d'instrumentalisation de la nature par la

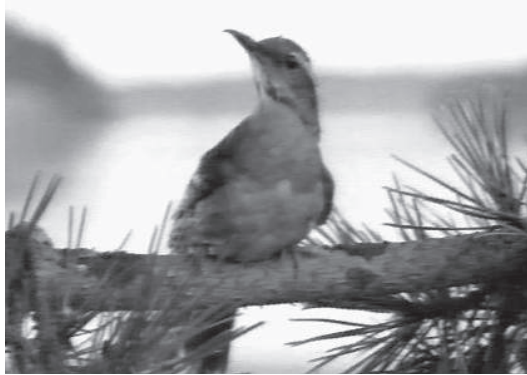

1

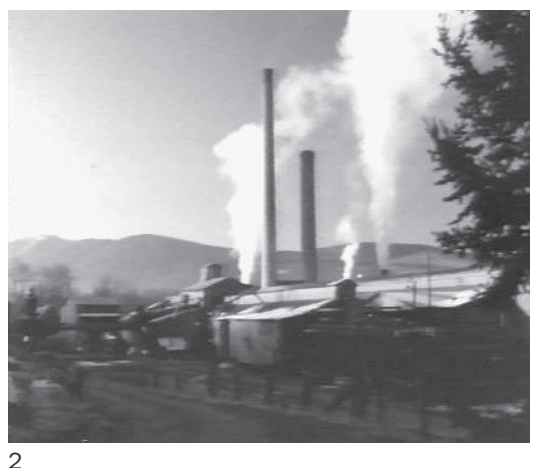


technique, que l'évacuation de tout opérateur humain à l'intérieur de l'aciérie renforce encore. L'oiseau au seuil du générique tend à acquérir au fil des épisodes un statut de logotype (en lieu et place du lion rugissant de la MGM, si l'on peut oser le parallèle), et renvoie à la série culturelle du transcendantalisme américain ${ }^{2}$. Le monde vert, une fois mis en chaîne avec le monde industriel, apparaît comme le lieu où l'on peut se ressourcer et restaurer une harmonie primitive entre l'homme et son environnement naturel (et ce n'est pas un hasard si, par la suite, l'agent du FBI surinvestit d'espoir de résolution du meurtre un oiseau bien nommé Waldo ${ }^{3}$ qui a assisté aux derniers instants de Laura Palmer, et qui sera abattu pour cette raison même). Paradoxalement, les plans de détail sur les rouages des mécanismes de l'usine (fig. 3-4) participent de cette harmonie: la régularité du mouvement de leur engrenage tend à les assimiler au cycle du monde naturel; et comme le confirme l'intrigue de la série, c'est bien l'aciérie qui est menacée par l'extension du complexe hôtelier du Great Northern. Ce jeu d'interversion entre la valence positive et négative des signes est décliné par la suite: la forêt, qui est appelée à jouer un rôle central dans Trin Peaks, est le lieu de toutes les menaces; le mal, qui s'avère omniprésent, est incarné par un avatar de l'homme des bois (Bob, l'esprit qui investit les hommes et qui a l'apparence d'un «hippy vieillissant» $\mathbf{4}$. Inversement, l'écologisme et le primitivisme vont aller croissant dans le discours de Ben, le propriétaire du Great Northern, et dans son mobilier qui multiplie les signes d'appartenance à une culture totémique.

L'antépénultième plan du générique nous positionne explicitement à l'entrée de Twin Peaks (fig. 5). L'image est en perspective, mais procure une forte impression de platitude: le panneau publicitaire duplique sous une forme schématisée le paysage dans lequel il s'inscrit; celui-ci paraît rabattu au premier plan. L'image qui donne à voir l'aube (chaque épisode de la série débute au lever du jour et se termine à la tombée de la
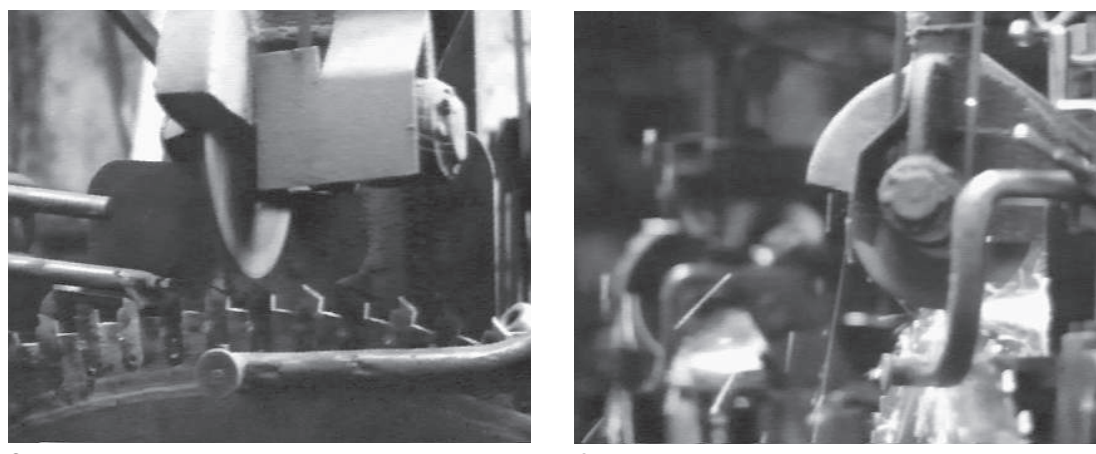

2 Nous pensons bien évidemment aux textes de philosophes et écrivains de la nature du $X I X$ siècle, tels que Ralph Waldo Emerson, Henri David Thoreau et Walt Whitman.

3 Ralph Waldo Emerson point à l'horizon - tout comme Laura (Otto Preminger, 1944), un personnage nommé Waldo Lydecker et renvoyant au Pygmalion de Laura, apparaissant plus tard dans la série.

4 Michel Chion, David Lynch, Editions de l'Etoile / Cahiers du Cinéma, Paris, 1992, p. 128. 


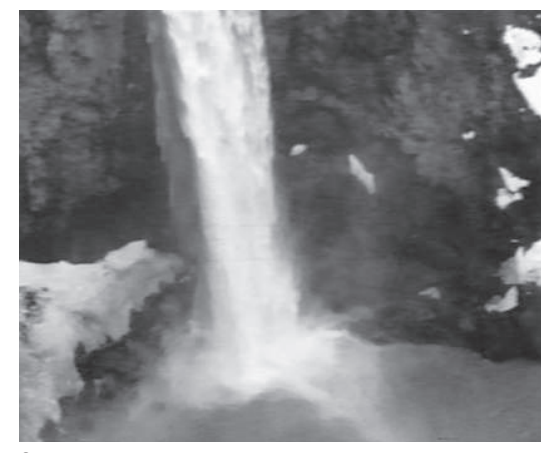

6

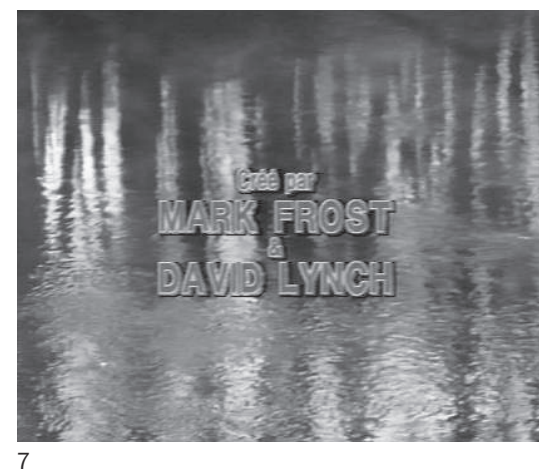

5 De la réalisation architecturale effective de Frank Lloyd Wright, Hitchcock fera le repère nocturne de la bande de trafiquants de secrets dans North by Northwest (La mort aux trousses, 1959).

6 Cf. Martin Heidegger, "La question de la technique" [1953], in Essais et conférences, Gallimard, Paris, 1958.

$7 \mathrm{Ce}$ motif macabre est à l'origine du titre du fanzine entièrement dévolu à la défense et à l'illustration de la série, Wrapped in Plastic.

8 Significativement, le film embraye alors sur la représentation du lycée de Twin Peaks, fréquenté par des jeunes filles innocentes - ou, tout au moins, arborant les signes les plus voyants de l'innocence (socquettes blanches, jupes sévères et, pour toute audace, quelques minauderies et intrigues de midinettes). Le lycée, dans la série, n'avait d'autre fonction que d'arrière-plan: nous n'y pénétrions que tardivement et à la faveur d'un coup de force scénaristique, en suivant un personnage retombé en enfance (Nadine Hurley) à qui les portes de l'établissement s'ouvraient pour des raisons thérapeutiques.

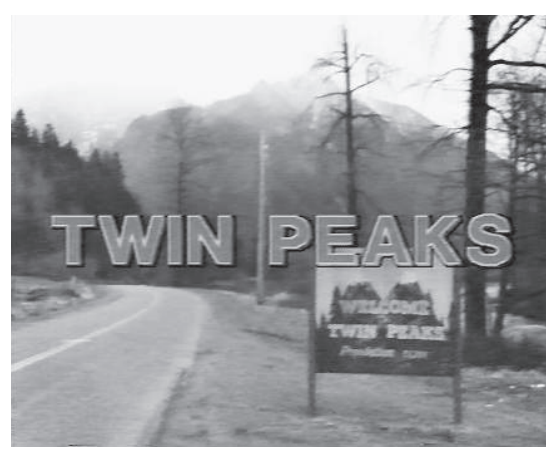

5 nuit) a tout de la "peinture idiote", entrée en matière qui n'est pas pour nous étonner au seuil d'une première "saison" en enfer (correspondant aux huit premiers épisodes).

Le motif de la cascade (fig. 6-7), renvoyant à la tradition romantique de la peinture de paysage où l'homme est réduit à portion congrue, intègre à travers les panoramiques de la caméra le mouvement qui, jusqu'alors, était interne au plan. Le complexe hôtelier du Great Northern, évacué d'entrée de jeu par un plan qui accompagne le mouvement de la chute d'eau (fig. 6), reste tapi dans le hors champ jusqu'au plan inaugural du premier épisode. La maison près de la cascade 5 entre par la suite dans une relation antagoniste avec l'aciérie. Cette dernière, située en contrebas de la cascade, canalise l'eau pour en tirer son énergie: le montage suggère un "arraisonnement techno-scientifique de la nature» $\mathbf{6}$, sans pour autant encore porter atteinte à l'harmonie du milieu. Le cours d'eau (fig. 7) participe sans ambiguïté à la série du monde vert apaisé - avant d'être à son tour instrumentalisé pour se débarrasser du corps de Laura Palmer, ce qui bien évidemment modifie rétroactivement le caractère idyllique du lieu. A travers un cadrage analogue au dernier plan du générique, le cadavre, emballé dans du plastique ${ }^{7}$,

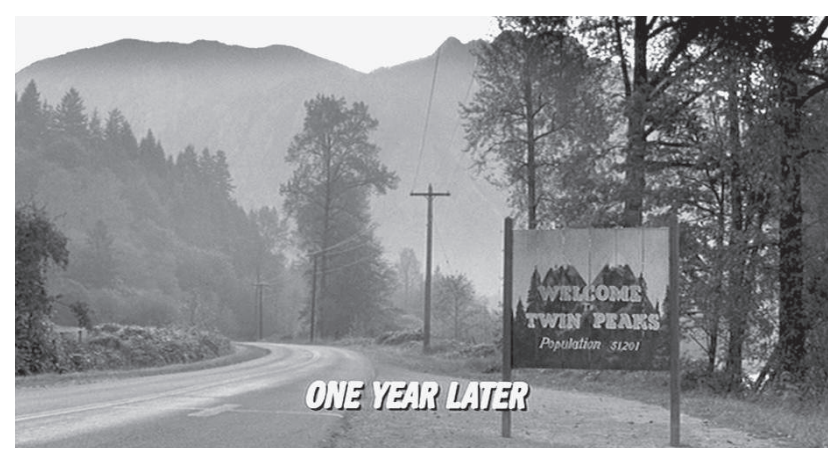

8 
dérive le long de l'eau - à l'image des déchets charriés par l'activité criminelle des hommes.

L'antépénultième plan du générique de Troin Peaks est repris dans le film dérivé de la série, Fire Walk With Me (1992): il est brièvement cité après trente-deux minutes, en position de clôture d'un prologue qui s'avère détaché aussi bien de la diégèse du film que de celle de la série. Sa fonction est uniquement intertextuelle: le plan, avec son adresse de bienvenue au visiteur (au spectateur), intervient comme un point de relais entre la série et le film. Celui-ci, en remontant en amont dans la chronologie de l'histoire, documente la vie de la communauté de Twin Peaks avant la mort de Laura Palmer (qui a déjà eu lieu quand débute la série). Fire Walk With Me se donne donc comme une sorte de pre-sequel qui supplémente la série, sans pour autant lui conférer davantage d'épaisseur narrative ou de vraisemblance. Les inscriptions graphiques soulignent le jeu de l'analepse: dans la série, le titre "Twin Peaks» apparaît dans la partie supérieure du cadre (fig. 5); dans le film, la mention "One year later", s'inscrivant au bas du cadre (fig. 8), signale une période temporelle intercalaire - le plan de situation intervient avant les événements de la série mais après ceux du prologue 8 . La mention «Un an plus tard» contribue à rejeter dans un passé de plus en plus éloigné le prologue qui, dans sa duplicité, embraye et enraye la narration du film, tout en débrayant celle de la série.

\section{L'extraction du spectateur hors de la fiction}

Le générique de Fire Walk With Me se déploie sur le fond d'une image abstraite (fig. 9) qu'un travelling arrière ancre rétrospectivement dans l'univers diégétique: il s'agit en effet d'un écran neigeux (et bleuté) de télévision, filmé plein cadre (fig. 10). Une fois identifié, le poste de télévision explose sous les coups redoublés d'un instrument qui n'apparaît que fugacement, les hurlements d'une femme ponctuant la scène (fig. 11) ; l'agresseur passe alors devant le poste télévisé, instaurant ainsi le noir à l'écran (fig. 12). Un fondu à l'ouverture découvre un cadavre qui dérive au fil d'eau (fig. 13). Celui-ci est identifié par une mention graphique qui se superpose au plan et dont le lettrage est identique à celui du générique : il s'agit du personnage de Teresa Banks (et non de l'actrice qui l'incarne), au meurtre duquel nous venons d'assister - ce que nous ne comprendrons que par la suite.

L'inscription liminaire du dispositif télévisuel dans le film renvoie au mode de diffusion de Trein Peaks - qui constitue par ailleurs le seul trait distinctif de la série vis-à-vis de la pratique filmique (rappelons que chaque épisode, tourné en support pellicule et supervisé selon
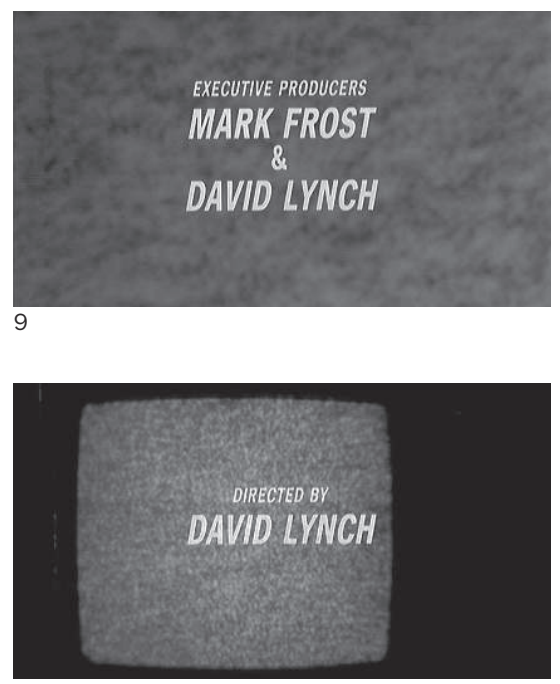
10

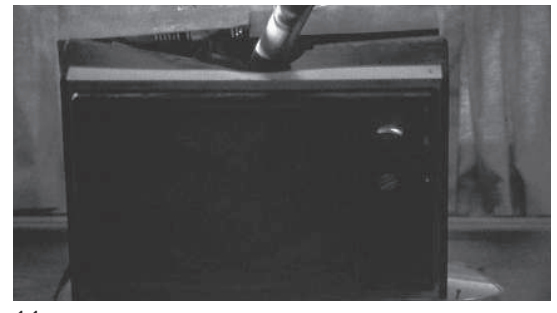

11

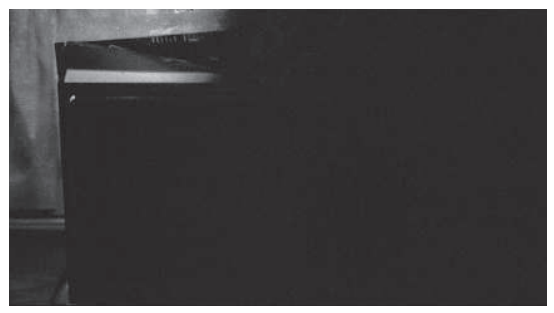

12

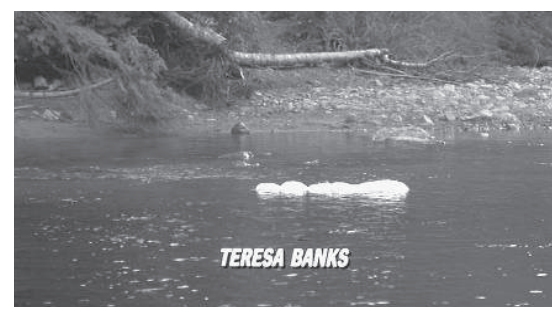


les standards cinéma, coûte 900000 dollars). En une métaphore prise à la lettre, l'explosion du poste TV annonce l'implosion des éléments fictionnels de la série, recombinés sur un mode elliptique à l'échelle de l'univers filmique. Car Fire Walk With Me, malgré son régime intertextuel, neutralise l'univers télévisuel par le biais d'une dynamique de recouvrement et d'effacement - qui n'est pas sans faire penser à des procédures que Lynch mettra en jeu à l'intérieur d'un même film (Lost Highway, 1997, Mulholland Drive, 2001). Aussi le passage au noir, d'abord diégétisé (le meurtrier passe devant le poste TV), puis reversé au compte d'une marque conventionnelle de ponctuation (un fondu à l'ouverture), peut être interprété comme l'objectivation formelle de la transposition déceptive que le film va opérer vis-à-vis de la série.

Accusant l'extraction hors de l'univers télévisuel, les plans qui suivent le brutal incipit iconoclaste du film renforcent l'artificialité des images. Gordon Cole, affublé de son sonotone (interprété par David Lynch qui signe le film par son apparition - alors qu'il intervient d'abord sous le couvert d'une voix assourdissante dans la série), se découpe sur le fond d'une photographie de paysage comportant un plan d'eau (fig. 14). Un raccord dans l'axe en plan plus large met en situation ce décor traité en aplats (et plutôt invraisemblable pour un supérieur du FBI): Gordon Cole donne des ordres à sa secrétaire qui traverse l'espace du bureau et sort du cadre après avoir adressé un regard à la caméra. Le plan suivant, en extérieur, est tout aussi improbable: dans un décor naturel, dont l'artificialité est accusée par le traitement de la lumière, quatre agents du FBI reprennent la maîtrise d'un bus scolaire détourné par un homme et deux femmes (la prise d'otages étant ponctuée par des hurlements d'enfants). Cette scène de genre, ne respectant aucune règle en la matière, contrevient à tout effet de réel.

Ce mode d'exposition distancié et peu économique est thématiquement relayé par les obstacles qui font écran à la bonne transmission des informations. Ainsi, la mission des détectives est révélée à travers la sémiologie invraisemblable d'une collaboratrice du FBI : les informations nécessaires à l'enquête sont livrées à travers ses codes gestuels et vestimentaires qui relèvent du pur arbitraire - et qui ne sont intelligibles au spectateur qu'à travers leur verbalisation rétrospective par le détective à l'intention de son coéquipier. Cette parodie de la codification non verbale est prolongée par un jeu d'inversion systématique des éléments positivés dans la série, sur un mode caricatural et volontairement déceptif (l'accueil des autorités locales et de la population est hostile, les espaces de socialisation tels que le café sont rédhibitoires, etc.). Le prologue s'attache avant tout à dépersonnaliser et à dédifférencier la 
figure du détective. Dans la série, le personnage principal (Dale Cooper) est présenté comme une figure archangélique qui occupe la fonction de foyer de l'identification - ce qui ne l'empêche pas d'être in fine possédé par l'esprit du mal (rappelons que Troin Peaks ne prend fin que pour des raisons contingentes, liées à l'interruption de la série). Dans le film, Dale Cooper n'apparaît qu'à la toute fin du prologue. Réduit au statut d'employé ordinaire du FBI, il prend le relais d'une équipe de détectives qui l'ont devancé sur cette affaire. Parmi eux, un agent, Chet Desmond, disparaît, tandis qu'un autre, disparu depuis deux ans, Philip Jeffries, resurgit furtivement avant de quitter définitivement la scène. Lorsque le premier détective découvre la bague portée par Teresa Banks (un bijou serti d'un motif de scarabée égyptien qui désigne la prochaine victime de Bob), il disparaît au gré d'un arrêt sur image accompagné d'un fondu au noir. Une fois encore, un procédé technique est sémantisé (l'image gelée et le fondu littéralisent l'expression "passer de vie à trépas»).

Le film et la série répondent à des logiques génériques concurrentes. Le film intègre les événements surnaturels dans un cadre de référence naturel: les forces du monde extraterrestre se manifestent sans ambiguïté (in corpore) à l'attention des personnages hantés (Laura Palmer, Teresa Banks, Philip Jeffries). La série rapporte les événements surnaturels à la subjectivité des personnages : il ne s'agit d'abord que de visions fugitives (fantasmatiques) qui, par la suite, prennent corps dans un cadre de référence naturel par l'entremise des rêves de Cooper ${ }^{9}$. Le mode d'introduction (dépsychologisé) des éléments surnaturels dans le film ne peut que retenir notre attention: une image télévisuelle, qui demeure invisible dans le monde de la diégèse, se superpose à l'écran de cinéma, comme nous allons le montrer.

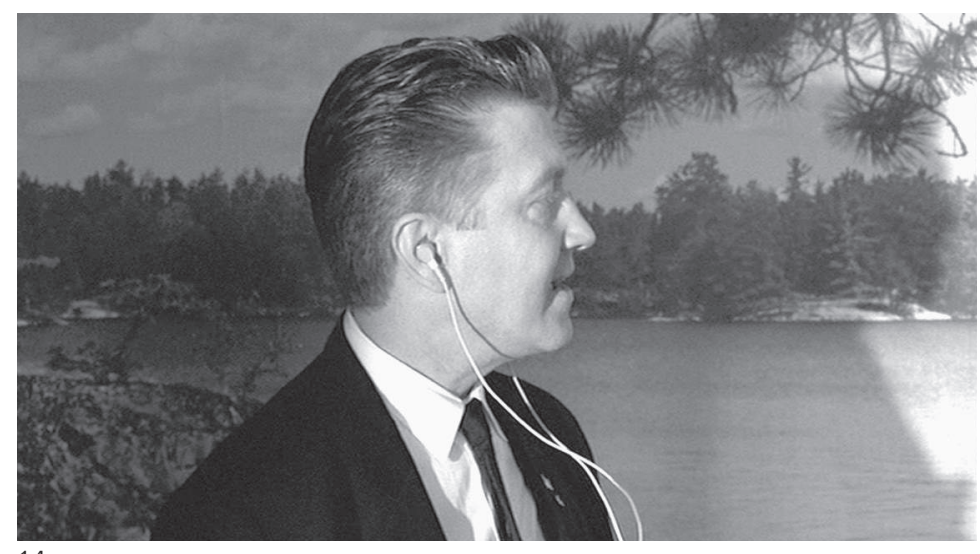

9 Diane Stevenson soutient que la série Twin Peaks repose sur le mode du fantastique, tel que Todorov l'a défini, pour introduire de façon médiate et distancée les éléments de l'inceste et de la violence familiale. Pourtant, la mise à distance de la romance incestueuse n'est pas suffisante pour prévenir un sentiment de déplaisir chez le téléspectateur: la révélation du criminel sous les traits de Leland Palmer a contribué à la chute du taux d'audience de la série. A notre avis, la série répond à un régime mixte qui ne met pas en jeu l'ambivalence énonciative du fantastique: la première saison est à dominante étrange (les codes de l'enquête policière, avec son effort de rationalisation, sont respectés); la seconde est à dominante merveilleuse (les éléments horrifiques sont désamorcés, les forces surnaturelles sont acceptées comme naturelles). Le film, en maintenant un cadre de référence naturel, ressortit plutôt au mode paradoxal du merveilleux horrifique. Cf. Diane Stevenson, "Family Romance, Family Violence, and the Fantastic in Twin Peaks", in David Lavery (éd.), Full of Secrets. Critical Approaches to Twin Peaks, Wayne State University Press, Detroit, 1995, p. 70-81. 
10 Pour suivre les méandres de ces récits à tiroir, nous renvoyons au découpage séquentiel de Twin Peaks et de Fire Walk With Me établi par David Lavery. Cf. David Lavery (éd.), Full of Secrets. Critical Approaches to Twin Peaks, op. cit., p. 208-258.

\section{Le dispositif de télésurveillance inséminé au centre du film}

Le prologue de Fire Walk With Me se distingue, on l'a vu, par la radicalité de son mode d'ouverture qui dénie violemment le dispositif télévisuel. Ce dernier est pourtant réintroduit par la suite: un écran neigeux de télévision, qui n'est visible que pour le spectateur, constitue la marque d'énonciation des événements surnaturels. L'image télévisuelle (niée - mais le pas qui tient à distance la négation de l'abstraction est vite franchi) apparaît donc comme le mode de monstration qui préside à la logique narrative du film. Mais il y a plus : le dispositif de la caméra de télésurveillance, qui est introduit lors d'une séquence décisive, forme le trope privilégié du film.

La caméra de télésurveillance, en authentifiant la communication entre les mondes naturel et surnaturel, apparaît comme l'opérateur formel de mise en contact d'univers parallèles: c'est à travers l'enregistrement d'une caméra reliée à trois postes TV que Dale Cooper surprend une trace indéniable du passage de l'agent disparu Philip Jeffries (sur un poste, l'image de Dale Cooper se fige et laisse apparaître Philip Jeffries dans le champ; Dale Cooper et Gordon Cole visionnent à nouveau la scène après que le détective s'est définitivement évaporé dans la nature). La séquence suivante, qui se signale par la surimpression récurrente d'un écran neigeux, a pour fonction de condenser les découvertes et les expériences du détective disparu. Une série de plans fantasmatiques retracent, sans la médiation du dispositif de télésurveillance, le parcours de Philipp Jeffries et mettent en scène des entités surnaturelles qui, dans la série, étaient ancrées dans la subjectivité onirique de Laura Palmer et de Dale Cooper. Tout se passe comme si, de la série au film, puis au sein même du film, l'investissement de la surface écranique par des mondes parallèles s'autonomisait de plus en plus (sans plus avoir recours à la médiation de personnages ou de dispositifs visuels diégétiques). L'écran fonctionne ainsi comme une membrane absorbante: il présente d'abord les personnages appelés à jouer un rôle central dans le déroulement des événements, puis escamote Philip Jeffries via la superposition d'un écran neigeux et de fils électriques. Le parcours initiatique du Dale Cooper de la série est ici récapitulé à travers un jeu de superposition des différentes couches de récit que la série dépliait et dilatait quant à elle. L'univers du film, à l'instar de certaines stratégies de désublimation (ou de désubjectivation) du pop art, prend comme seul cadre de référence la série, en en redistribuant les motifs et les intrigues $\mathbf{1 0}$.

En effet, la série est construite sur le principe d'une infinie ouverture des fils du récit, de ses rebondissements et de ses dénouements. Le canevas de base change abruptement à plusieurs reprises: les personnages, 
leur psychologie et les liens qu'ils nouent entre eux sont soumis à des procédures de réécriture; le régime générique de la série ou, si l'on préfère, son pacte de lecture ne respecte pas le principe de non-contradiction. Fire Walk With Me, considéré dans son autonomie discursive, constitue un remarquable enchevêtrement de motifs, de personnages et de situations narratives qui résistent à toute linéarisation et psychologisation des événements. Par contre, une fois rapporté à son texte source (la série Twin Peaks), le film (à l'exception de son prologue) s'apparente à un exercice formel quelque peu vain, se situant nettement en deçà de l'ouverture et de l'irrésolution des fils narratifs de la série, de l'invraisemblance et de l'arbitraire de situations qui échappaient à la mainmise d'un auteur unifié.

Passons outre, et énumérons les différentes occurrences de l'écran neigeux "dédiégétisé», ou parfois de flashs bleutés qui introduisent, au-delà du prologue, les personnages et les événements issus de mondes parallèles. La famille Chalmont apparaît à Laura Palmer par le biais d'un champ/contrechamp - la surimpression d'un écran neigeux attestant leur appartenance au monde surnaturel. Le tableau qu'ils offrent à Laura se révèle être une clef d'accès à l'autre monde: Laura pénètre au sein de la représentation picturale; guidée à travers une enfilade de portes par les Chalmont, elle fait irruption dans le rêve de Cooper. Le tableau, qui reflète comme à travers un miroir l'espace de la chambre de Laura, propose une alternative à la représentation télévisuelle: le mode de la contemplation ou de l'absorption dans la représentation picturale, ici littéralisé, acquiert une fonction transitionnelle. La bague constitue un autre lieu de passage: en contrechamp à des travellings avant sur Laura, des images souvenirs, reliées par la récurrence du motif de la bague, surgissent (des personnages surnaturels ou décédés : le manchot et le nain, Teresa Banks sont introduits par une lumière électrique intermittente). Parallèlement, dans le salon des Palmer, une image neigeuse plein cadre interrompt le plan de Leland Palmer et laisse place à une visualisation du meurtre de Teresa Banks.

La lumière bleue intermittente est associée à l'apparition de Bob, l'esprit maléfique, et aux lignes électriques: c'est par son entremise que l'on découvre l'identité cachée de Leland Palmer et que l'on assiste au meurtre de Laura dans le wagon (par le biais du montage, une identité trouble est suggérée entre Laura, le personnage du nain et Bob; et, à la faveur d'un jeu de reflet, le visage de Bob se substitue à celui de Laura dans le miroir). Relevons encore que les occurrences de flashs bleutés peuvent à l'opposé valoir comme signe de la déité et comme annonce d'une apparition archangélique: ainsi lorsque, pendant la séquence du 
11 Propos rapportés par Michel Chion, David Lynch, op. cit., p. 122. meurtre, Ronette Pulaski implore Dieu, ses liens se délient comme sous l'effet de cette lumière bleutée; et le plan final, lourdement souligné par le Requiem en ut mineur de Luigi Cherubini, propose comme apothéose la rédemption de Laura Palmer sous les feux des sunlights. Cette clôture très marquée n'est pas sans contrevenir à la logique de la série et au modèle, jusqu'alors relativement respecté, du texte palimpseste où un nouveau tracé signifiant se superpose aux inscriptions antérieures sans pour autant les condamner à l'inexistence. Il n'est peut-être pas illégitime de pronostiquer une provocation de la part de Lynch qui met un terme, sur le mode hollywoodien, à un projet télévisuel qui pouvait théoriquement se poursuivre à l'infini...

\section{La télévision comme aire libre de jeu...}

Lynch est on ne peut plus explicite au sujet de la structure digressive et de l'infinie ouverture du jeu que permet la série: "L'idée de continuité à la télévision est formidable. Ne jamais dire au revoir», déclarait-il dans les pages de Libération 11. Et de fait, la fin de Twin Peaks est contingente: l'investissement de Dale Cooper par l'esprit de Bob ne constituait qu'un gimmick qui devait relancer les péripéties. La diffusion tourmentée de la série, suspendue entre la première et la deuxième saison, en a décidé autrement: la défection du public a provoqué son interruption abrupte. Ainsi, si Trein Peaks a immédiatement donné lieu à un phénomène de culte, l'hétérogénéité des horizons d'attente générique et des contrats de lecture mis en jeu a rapidement découragé le public. Les premiers épisodes sont structurés selon les codes du film policier, entrecroisés avec les conventions du soap opera. Dès que l'énigme policière est résolue et la situation incestueuse révélée, ce premier horizon d'attente générique est suspendu. Intervient alors le modèle du merveilleux, associé à un jeu de détournement parodique : le premier horizon d'attente générique de la série fait l'objet d'un pastiche généralisé. La pluralité des codes de structuration du récit permet de jouer à l'envi avec les intertextes cinématographiques qui prolifèrent dans la série. Nous pouvons tenter de catégoriser cursivement différents modes d'activation intertextuelle.

Un premier mode consiste en l'activation d'un seul code générique. Il s'actualise généralement à travers un épisode doté d'un fort degré d'autonomie narrative qui a une fonction de digression. Ainsi, les codes du film noir donnent-ils lieu à un épisode comme enchâssé qui suspend provisoirement le déroulement de l'intrigue principale: la thématique de la manipulation d'un homme par une femme fatale, marquée par un déplacement géographique, y est déclinée volontairement ad nauseam. Des films tels que Double Indemnity (Billy Wilder, 1944) ou The Postman 
Always Rings Treice (Le facteur sonne toujours deux fois, Tay Garnett, 1946) sont mobilisés lors de cette incise narrative qui n'excède pas les conventions du genre et qui reconduit de façon délibérément plate les étapes obligées de cette instrumentalisation.

Un deuxième mode consiste en l'activation d'une pluralité de codes génériques qui entrent dans un rapport de coexistence pacifiée échappant à toute hiérarchisation. On est alors le plus souvent confronté à des références ponctuelles, localisées et aisément reconnaissables. Ainsi, l'arrivée de Leo Johnson dans la tanière d'un Windom Earle surgi du passé de Dale Cooper fait clairement référence, sur le mode de la subversion, à une scène du premier Frankenstein (James Whale, 1932), archétype du fantastique scientifique pratiqué par la Universal des années 1930. Néanmoins, cette situation donne lieu à toute une série de références aussi bien cinématographiques (principalement au film criminel) qu'extra-cinématographiques (essentiellement au merveilleux reinhardtien).

Un troisième mode consiste en la citation d'un film ou d'une série TV liés à un genre bien défini. L'exemple type, sur le plan cinématographique, demeure la référence à The Exorcist (L'exorciste, William Friedkin, 1973) lors des scènes de possession de Laura Palmer par Bob. Dans le champ télévisuel, le soap opera fait l'objet d'un pastiche, certains personnages regardant une série intitulée Invitation to Love. La disparition de cette série se fait au profit d'une contamination intermittente de la fiction de Twin Peaks par l'univers du soap: ainsi, lorsque Lucy doit se déterminer entre deux pères pour l'enfant qu'elle porte, la série s'ouvre au retour régulier des codes du mélodrame à l'eau de rose, avec une indéniable intention parodique.

Un quatrième mode consiste en l'accumulation des effets de style marqués "cinéma» ou "série TV». L'exemple le plus remarquable du côté "cinéma» en est l'épisode 22, tourné par Uli Edel, qui abuse de courtes focales et de plongées accentuées et qui mobilise profondeur de champ et éclairages travaillés. A suivre la démonstration de Michel Chion 12, les épisodes tournés par David Lynch lorgnent volontairement du côté de l'effacement de l'auteur en se cantonnant dans une esthétique télévisuelle.

On peut remarquer que la pluricodicité générique va s'accentuant au fil des épisodes, notablement après la révélation du meurtrier de Laura Palmer: une dimension parodique s'installe alors, neutralisant tout positionnement affectif univoque du spectateur; la prévalence de la tonalité horrifique de l'univers de la série est suspendue, minimisant l'impact des forces souterraines et rejetant la situation de l'inceste dans le passé de la série. Cette mise entre parenthèses peut affecter la fonction narrative 
13 A suivre la catégorisation des logiques virales que propose Susan Sontag, nous pouvons soutenir que la majeure partie de la seconde saison de Twin Peaks répond à la logique des imprévisibles mutations du sida plutôt qu'à celle de la contamination progressive des cellules saines par le tissu cancéreux. Cf. Susan Sontag, La maladie comme métaphore. Le sida et ses métaphores, Christian Bourgois Editeur, Paris, 1993 [1977/1988]. des personnages. Ainsi, l'identité de Ben Horne est sujette à un revirement spectaculaire: ce père aux tendances incestueuses, propriétaire d'un lupanar transfrontalier et, surtout, représentant de l'adaptation du milieu naturel au tourisme industrialisé, devient un écologiste convaincu et arbore tous les signes d'un primitivisme amérindien - après avoir rejoué (sur un plan fantasmatique et thérapeutique) la guerre de Sécession au profit du Sud. De même, les relations entre personnages peuvent s'inverser radicalement: le shérif local Harry Truman et le médecin légiste citadin Albert Rosenfeld, tout d'abord ennemis naturels et invétérés, finissent par se donner l'accolade et répondre à l'unisson. La musique peut accompagner ces renversements: les leitmotivs circulent d'une situation à une autre et transitent d'un personnage à un autre.

Somme toute, cette logique dispersive en termes de genres, de positionnement spectatoriel, de schémas narratifs et de modes de représentation légitime la désaffection du public: la série renonce, après la première saison, à l'activation simple et sur-référenciée, avec ou sans visée subversive, des codes télévisuels et cinématographiques pour se laisser contaminer par une logique à proprement parler sérielle. Celleci peut être caractérisée comme une variation autour de schémas qui sont explorés, si ce n'est jusqu'à leur épuisement, tout au moins jusqu'à leur dysfonctionnement et leur ouverture à un mode concurrent de structuration. La série, en renonçant aux codes qu'elle a mis en place, résiste à la tentation de faire système et de le reconduire d'un épisode à un autre: elle prend systématiquement le contre-pied d'une logique classique de fidélisation d'un public formé à son usage. Dès lors, elle manifeste une remarquable ouverture de son jeu, en réinventant à tout instant ses règles. Selon nous, l'intervention de réalisateurs et de scénaristes extérieurs au team de production Lynch-Frost, quand bien même a-t-elle pu être plus ou moins planifiée d'avance, a permis de relancer la logique de la série: Lynch s'éclipsant pendant près de quinze épisodes, les différents intervenants sur la série ont su instaurer un mode narratif qui se caractérise par le flottement des péripéties et l'étirement des fils de l'intrigue; à l'image du virus du mal (Bob) qui contamine sans distinction les corps porteurs, les derniers épisodes de Trwin Peaks répondent à d'incessantes mutations $\mathbf{1 3}$.

\section{... et jeu libre de langage}

Au fil des épisodes, l'intrigue de Trwin Peaks vole en éclats ou, tout au moins, se délite, suivant d'une part un mouvement de désamorçage de la raison, d'autre part un mécanisme de volatilisation du mal. Les codes de la série criminelle (surtout présents dans les épisodes 1 à 8) cèdent 
la place au fantastique (principalement dans les épisodes 9 à 15), mode à son tour dérouté par l'intrusion du merveilleux (autour des épisodes 16 à 30). L'effort de rationalisation du récit déchoit en pure et simple ratiocination qui, à son tour, bascule dans l'irraison. La série s'ouvre sur la traque policière d'un coupable, par le biais du recoupement d'indices confondants (encore que les méthodes d'investigation de Dale Cooper - qui s'en remet à ses rêves et à la sagesse tibétaine - confinent à l'irrationnel) : qui a donc tué Laura Palmer (ou encore, comme la communauté d'internautes fascinés par la série l'abrégeait, «WKLP»: «Who killed Laura Palmer»14)? Sous les pressions réitérées des commanditaires, c'est-à-dire la chaîne $\mathrm{ABC}$, la production Lynch-Frost était censée identifier le coupable dès le premier épisode de la seconde saison 15. La production fait alors astucieusement intervenir l'ambivalence énonciative du mode fantastique, mais pour aussitôt le secondariser: tout suspect, étant habité par la part d'ombre qui divise le sujet (le mythe du Dr. Jekyll et de Mr. Hyde est ici pris au pied de la lettre), est désigné coupable; mais les éléments inquiétants, les forces sourdes et extra-normales sont projetés au sein même de la cellule familiale - le monde parallèle n'est finalement qu'une projection de Laura Palmer, refoulant l'inceste qu'elle a subi ou peut-être bien fantasmé. Enfin, toute pression relâchée, le coupable est identifié à une menace extra-terrestre, où le territoire de la commune de Twin Peaks (pourtant privilégié, si l'on en croit les dessins cryptiques et les cartes astrales qui orientent les investigations de Cooper), apparaît comme un échiquier trop étroit pour jouer la partie en cours (rappelons que Dale Cooper et son ancien partenaire Wyndom Earle communiquent par échiquiers interposés, en transposant leurs coups dans le réel). Symptomatiquement, dans les premiers épisodes, Bob n'apparait qu'exceptionnellement en situation de coprésence avec d'autres personnages dans le champ; par la suite, il se superpose, à travers des jeux de reflet, à des personnages qu'il contamine, comme dans le modèle des Vanités; et, enfin, il se volatilise pour emporter, à travers l'éclat d'un flash de lumière blanche, le major Briggs, incarnation caricaturale d'une raison néanmoins déréglée par l'observation d'activités extra-terrestres.

Quoi qu'il en soit, la série Trwin Peaks se caractérise par un jeu de combinaison infinie de pièces qui peuvent tour à tour s'emboîter et se défausser. Le rapport entre la série, décidément centrifuge, et le film, résolument centripète, est donc diamétralement opposé: si le film se subordonne (diégétiquement) à la série qui, par une infalsifiable sortie de bande, part à la déroute, c'est pour mieux donner une cohésion aux événements qui étaient jusqu’alors disséminés ou suspendus. La reprise
14 Cf. Henri Jenkins, " 'Do You Enjoy Making the Rest of Us Stupid?,: alt.tv.twinpeaks, the Thrickster Author, and Viewer Mastery", in David Lavery (éd.), Full of Secrets, op. cit., p. 51-69.

15 Cf. Stefan Peltier, "Twin Peaks, un protagoniste en série", in Eclipses, n 34, 2002, p. 100. 
16 Cf. Gilles Deleuze, Felix Guattari, Rhizome, Editions de Minuit, Paris, 1976; Gilles Deleuze, L'image-mouvement, Editions de Minuit, Paris, 1983.

17 Cf. Max Horheimer, Theodor W. Adorno, "La production industrielle des biens culturels", La dialectique de la raison [1944], Gallimard, Paris, 1974; Theodor W. Adorno, "Transparents cinématographiques " [1966], in Pratiques, $n^{\circ} 14$, automne 2003 auteuriste d'un écheveau qui se dévidait, la maîtrise forclose d'un jeu dont les règles n'étaient pas fixées mettent terme à la logique combinatoire et aléatoire de la série. En empruntant une terminologie deleuzienne, nous pouvons soutenir que le film contrecarre la structure "rhizomatique» de la série en restaurant les «liens sensori-moteurs de l'action»16. Ce phénomène de reprise et de forclusion en vient d'ailleurs à se constituer en véritable marque de style et de signature dans le système lynchien. A notre sens, les réalisations de Lynch ultérieures (et, pourquoi ne pas le dire, antérieures) à Trein Peaks répondent à un mouvement similaire: la multiplicité des fils et l'ouverture des interprétations exhibent une fausse complexité narrative qui est brandie pour produire une impression d'ambiguïté ou un effet d'équivoque. Lost Highrway et Mulbolland Drive (et dans ce dernier cas, le film réordonne le pilote d'une série qui n'a pu être tournée) reposent sur un texte cohésif qui ordonne une structure dispersive, en se conformant à la position de maîtrise de l'auteur qui programme une perte de contrôle de la narration. Le texte filmique est, certes, ouvert, mais ses fils sont canalisés et ses motifs demeurent stables: Lynch, en reproduisant une logique narrative qui s'inaugure dans le rapport de Fire Walk With Me à Troin Peaks, exhibe sa capacité à réécrire le même film sous une nouvelle forme, en redistribuant les rôles et le dessin de l'intrigue tout en maintenant une maîtrise et une mainmise sur la forme narrative. La dispersion est régulée et instrumentalisée : la reprise auctoriale des fils du récit mime l'éclatement et la dispersion de la structure du film sans la mener à son terme.

Lynch réalisateur de films qui exhibent leur inquiétante étrangeté et Lynch coproducteur d'une série télévisée dont le jeu peut a priori se poursuivre à l'infini répondent à des stratégies inverses: Twin Peaks annonce rapidement, pour des raisons promotionnelles, la résolution de sa trame narrative qui finit par voler en éclats; Lost Highway et Mullholand Drive, empruntant tous deux une forme sérielle, annoncent un mouvement de dissémination du récit sans tenir leur promesse. Au risque de pêcher par excès d'anti-auteurisme, nous affirmerions volontiers que la série télévisée présente une aire libre de jeu que la production Lynch-Frost investit, en se déployant sous forme de jeux libres de langage. Souvenons-nous de la critique radicale que Theodor W. Adorno et Max Horkheimer portent à l'encontre de l'industrie du divertissement, et du tardif revirement de position d'Adorno qui peut écrire en 1966, quatre ans après que le manifeste d'Oberhausen (contre le "cinéma de papa») a été signé, que l'industrie culturelle "possède l'antidote à son propre mensonge»17. Adorno, rappelons-le, adhère alors à la position, défendue par Oskar Negt et Alexander Kluge, d'une «sphère publique 
de résistance» $\mathbf{1 8}$. Seulement, si l'on peut accorder un tel crédit à la série produite par Lynch et Frost, il semble bien que c'est l'anonymat de l'industrie télévisuelle qui a conduit à une prolifération de voix discordantes qui, dans le système auteurisé de la production filmique, étaient contenues par l'expression d'un point de vue unifiant. La conclusion de l'article d'Adorno, si nous excluons son ultime concession au négativisme de la théorie critique, peut selon nous s'appliquer au rapport entre série et film dans le système lynchien:

"Comme il serait beau de pouvoir prétendre, dans la situation d'aujourd'hui, que les films sont d'autant plus des œuvres d'art qu'ils s'affichent moins comme des œuvres d'art! On a fort envie de le faire, quand on est confronté à ces class A-pictures, sophistiqués et d'autant plus psychologiques, que l'industrie de la culture s'oblige à produire pour rehausser son image culturelle» $\mathbf{1 9}$.
18 La notion de "sphère publique de résistance " est élaborée par Jürgen Habermas, puis redéfinie et radicalisée par le sociologue Oskar Negt et le cinéaste Alexander Kluge, signataire du manifeste d'Oberhausen. Cf. Jürgen Habermas, L'espace public. Archéologie de la publicité comme dimension constitutive de la société bourgeoise, Payot, Paris, 1978 [1962]; Oskar Negt, Alexander Kluge, Öffentlichkeit und Erfahrung. Zur Organisationsanalyse von bürgerlicher und proletarischer Öffentlichkeit, Suhrkamp, Francfort-sur-le-Main, 1972.

19 Theodor W. Adorno, "Transparents cinématographiques ", op. cit. 